\title{
Getting back to points of origin
}

\section{A new analytical strategy allows researchers to map origins of replication within long stretches of genomic DNA.}

Since the sequencing of the human genome, a great deal of energy has gone into efforts to sequence genomes from a diverse array of other species. A big part of the motivation behind this effort is in the simple fact that direct comparison of sequence conservation over evolutionary distances is one of the most powerful tools for structural and functional genomic analysis.

Unfortunately, comparative analysis has proven inadequate for identifying one key genomic feature-mammalian origins of replication. "It is now more and more apparent that in higher eukaryotes... DNA sequence conformation around origins is very, very relaxed," explains Mauro Giacca, of the International Center for Genetic Engineering and Biotechnology in Trieste, Italy. "These regions bind to ORC proteins and several other members of the preinitiation complex, which are conserved from budding yeast to mammals, but probably the way they recognize the target is very different in mammalian cells in the sense that it's less sequence-dependent and more context-dependent." Currently, less than 30 human origins have been identified out of an estimated total of 30,000, and these have no apparent sequence similarity.

Over a decade ago, Giacca and his colleagues developed a method for the isolation and subsequent quantitative PCR amplification of nascent DNA from the replication bubble that forms at active origins (Giacca et al., 1994). Although this technique has proven relatively popular, it requires the generation of many closely spaced primer sets and is thus impractical for mapping origins in larger stretches of sequence. Now, in a new article from Molecular Cell, Giacca's group describes an expansion of the technique that promises to greatly simplify the gross mapping of origins within chromosomal regions of interest (Todorovic et al., 2005).
They begin by isolating small, size-selected fragments of nascent DNA from cultured cells, which are ligated with specialized linkers and PCR-amplified to generate a library of origin-enriched DNA fragments, which forms the foundation for a procedure that Giacca's group calls subtractive hybridization for origin capture (SHOC; Fig. 1) The library is depleted of repetitive sequences, and then hybridized against large fragments of biotinylated genomic DNA from a region of interest. Hybrids are isolated by streptavidin, and the selected clones are enriched via PCR amplification. The last stage of SHOC involves a comparative Southern hybridization experiment with restriction-digested genomic DNA, using duplicate filters that are probed either with unselected genomic DNA or the selected nascent DNA clones. By localizing preferentially hybridized fragments within the restriction map, it becomes possible to approximately map the location of active origins within a genomic region. These can then be finely mapped using quantitative PCR.

Using SHOC, Giacca's group identified at least two novel origins of replication from a stretch of chromosome 5. This initial study was only applied against a 50-kilobase genomic region, but Giacca believes this procedure could be scaled up considerably. "The future evolution is to use the same library as a probe against tiled microarrays, which would give [data for] entire chromosomes," he says. "We've already started a collaboration with the Sanger Center in the UK to set up this hybridization against tiled microarrays."

Even this early data has provided food for thought, however. As expected, the two newly identified origins appear to have no clear sequence relationship with previously characterized origins. There is, however, a 30base-pair (bp) stretch between the two origins that has a striking similarity to the 24-bp 'core' region of another, previously characterized origin. Giacca speculates that this 30-bp stretch could be important to solving the ori-

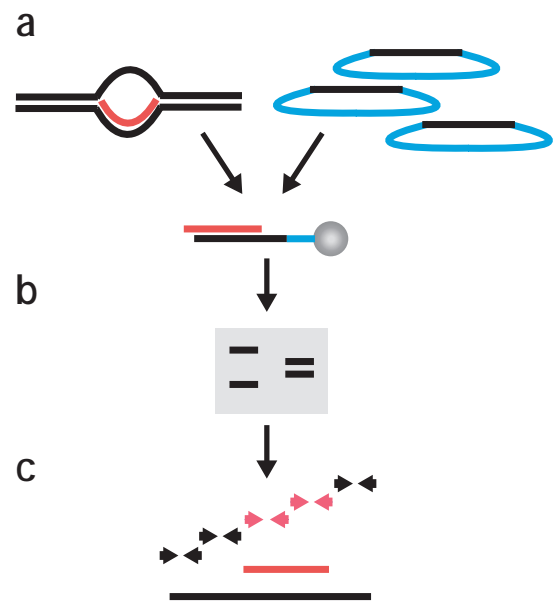

Figure 1 | I dentifying origins with SHOC. (a) Nascent DNA (red) is isolated and affinity purified by hybridization against streptavidin bead-bound cosmid clones (black). (b) Eluted DNA is amplified and used to probe restrictiondigested genomic blots, allowing crude origin localization. (c) Quantitative PCR of nascent DNA using primers against the region of interest (arrowheads) reveals location of origins.

gin puzzle. "We know that it binds something, because with in vivo binding studies based on footprinting, we see protection there," he says. "This could represent the conserved part of the origin, which is shared by all origins, while the actual start site for DNA replication, where we make the short stretches of DNA, are simply A-T-rich or easily meltable sites."

Giacca hopes that finding and studying additional origins will reveal originbinding factors that tie replication to other functions, such as cell cycle regulation. "What we'd like to do is to find new proteins that are not part of the prereplication complex that bind to origins," he says.

\section{Michael Eisenstein}

\section{RESEARCH PAPERS}

Todorovic, V. et al. Human origins of DNA replication selected from a library of nascent DNA. Mol. Cell 19, 567-575 (2005).

Giacca, M. et al. Fine mapping of a replication origin of human DNA. Proc. Natl. Acad. Sci. USA 91 7119-7123 (1994). 